\title{
Observações da dieta de Artibeus lituratus (Olfers) (Chiroptera, Phyllostomidae) em duas áreas do sul do Brasil ${ }^{1}$
}

\author{
Fernando C. Passos $^{2} \&$ Gustavo Graciolli ${ }^{2}$ \\ ${ }^{1}$ Contribuição número 1457 do Departamento de Zoologia, Universidade Federal do Paraná. \\ ${ }^{2}$ Departamento de Zoologia, Universidade Federal do Paraná. Caixa Postal 19020, 81531-980 Curitiba, Paraná, Brasil. \\ Bolsista do CNPq. E-mail: fpassos@ufpr.br; mingau@ufpr.br
}

\begin{abstract}
Diet observations of Artibeus lituratus (Olfers) (Chiroptera, Phyllostomidae) in two areas of southern Brazil. This study was carried out at Superagüi National Park (PNS) and Volta Velha Private Reserve of Natural Heritage (RPPN), both areas of Atlantic Rain Forest, Paraná and Santa Catarina states, in southern Brazil. Bats were mist netted and fecal samples were collected for dietary analysis. The seeds found in each sample were identified in the laboratory under stereoscopic microscope. A hundred and thirty seven Artibeus lituratus (Olfers, 1818) bats were captured ( $n=47$ in PNS, $n=90$ in RPPN), and 25 and 32 fecal samples were collected for each area, respectively. The frugivorous diet of Artibeus lituratus accounted for 81 to $88 \%$ of the total food itens showing the importance of fruit to this species.
\end{abstract}

KEYS WORDS. Atlantic Forest, bats, frugivory, seeds.

RESUMO. Este estudo foi realizado no Parque Nacional de Superagüi (PNS) e na Reserva Particular do Patrimônio Natural de Volta Velha (RPPN), ambas áreas de Mata Atlântica, nos Estados do Paraná e Santa Catarina, no sul do Brasil. Morcegos foram capturados em redes de neblina e amostras fecais foram coletadas para análise da dieta. As sementes encontradas em cada amostra foram identificadas em laboratório por meio de lupa. Cento e trinta e sete exemplares de Artibeus lituratus (Olfers, 1818) foram capturados ( $\mathrm{n}=47$ no PNS, $\mathrm{n}=90$ na RPPN), e 25 e 32 amostras fecais foram coletadas para cada área, respectivamente. A dieta frugívora de A. lituratus computou de 81 a $88 \%$ do total dos itens alimentares, mostrando a importância dos frutos para esta espécie.

PALAVRAS CHAVE. Frugivoria, Mata Atlântica, morcegos, sementes.

\begin{abstract}
A família Phyllostomidae, endêmica do continente americano, destaca-se como o grupo mais versátil na exploração de alimentos entre os Chiroptera, podendo explorar frutos, néctar, pólen, folhas, insetos, vertebrados e sangue entre outros. Entre as subfamílias, Stenodermatinae é composta principalmente por espécies frugívoras (Nowac \& Paradiso 1983). Uma de suas espécies de grande abundância e muito comum em áreas do sul e sudeste do Brasil é A. lituratus (Olfers, 1818). É um morcego frugívoro de grande porte, encontrado desde a região central do México até o sul do Brasil (Rio Grande do Sul) (Fabian et al. 1999, Rur et al. 1999). Sua dieta frugívora foi relatada por Fleming (1986), Galetti \& Morellato (1994), Marinho-Filho (1992), Sazima et al. (1994) e Zortea \& Chiarello (1994), mas outros recursos alimentares como insetos, folhas e néctar também são consumidos (FARIA 1995, Fischer \& FisCher 1995, SAZIMA et al. 1994, Zortéa \& Mendes 1993). O objetivo deste estudo foi realizar observações sobre a dieta de A. lituratus em duas áreas de Mata Atlântica da região sul do Brasil, nos Estados do Paraná e de Santa Catarina, para contribuir com um melhor entendimento sobre a ecologia alimentar dessa espécie.
\end{abstract}

\section{MATERIAL E MÉTODOS}

Este estudo foi realizado em duas áreas no litoral da Região Sul do Brasil (Fig. 1), ambas apresentando vegetação de Mata Atlântica, o Parque Nacional do Superagüi $\left(25^{\circ} 15^{\prime}\right.$ 'S$25^{\circ} 29^{\prime} \mathrm{S}, 48^{\circ} 06^{\prime} \mathrm{W}-48^{\circ} 19^{\prime} \mathrm{W}$ ), no município de Guaraqueçaba, Paraná, e a Reserva Particular do Patrimônio Natural (RPPN) de Volta Velha $\left(26^{\circ} 04^{\prime} 05^{\prime \prime} S, 48^{\circ} 37^{\prime} 30^{\prime \prime} \mathrm{W}\right)$, município de Itapoá, Santa Catarina. As coletas no Parque Nacional de Superagüi (PNS) foram realizadas na Ilha de Superagui, distante apenas algumas dezenas de metros do continente. Tais coletas foram nos meses de março, junho, setembro e novembro de 2001, durante aproximadamente cinco noites por mês, totalizando 106 horas de exposição das redes de neblina (mist nets). Em média as redes foram abertas por 5,3 horas por noite. No PNS as coletas foram realizadas em local próximo da Vila de Superagüi, em área de vegetação de Mata Atlântica de terras baixas. Já na RPPN de Volta Velha foram realizadas nos meses de maio, agosto, dezembro de 2001 e janeiro de 2002, também durante aproximadamente cinco noites por mês, totalizando 
108 horas de exposição. Em média as redes foram abertas por 5,4 horas por noite. Nessa área as coletas foram realizadas próximo da sede, caracterizada pelo mesmo tipo de vegetação que no PNS. Os morcegos retirados das redes foram acondicionados em sacos de algodão até o dia seguinte, quando as fezes foram coletadas e acondicionadas em tubos plásticos ("eppendorf"). Posteriormente, as fezes foram levadas para o laboratório, onde a limpeza e identificação da sementes foram realizadas. Finalmente, a análise da dieta de $A$. lituratus foi realizada através da freqüência de ocorrência dos recursos encontrados nas amostras de fezes.

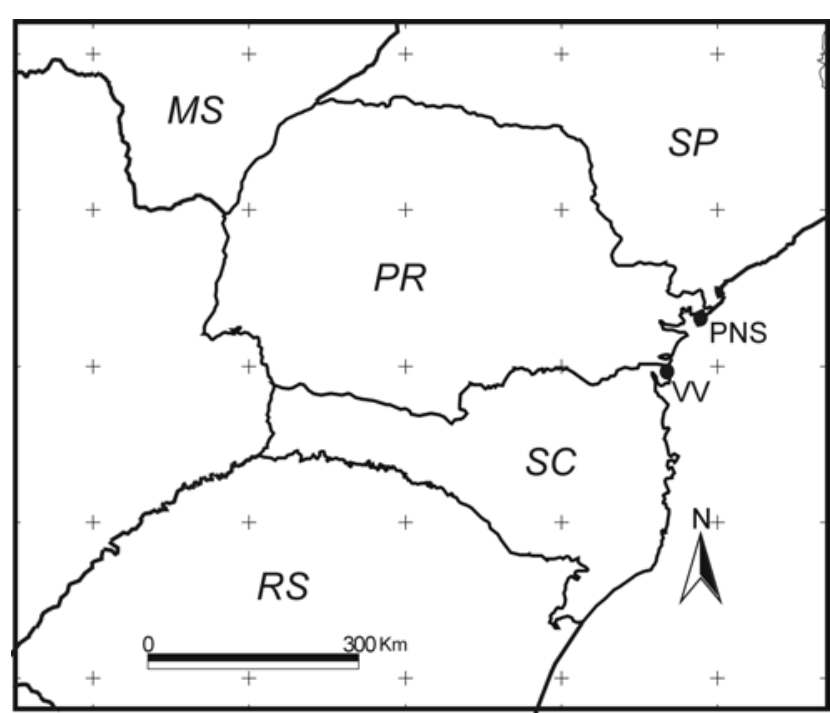

Figura 1. Localização das áreas de estudo na Região Sul do Brasil: Parque Nacional de Superagüi (PNS), Guaraqueçaba, Paraná e Reserva Particular de Proteção Natural de Volta Velha (RVV), Itapoá, Santa Catarina.

\section{RESULTADOS}

Foram capturados no Parque Nacional do Superagüi 47 indivíduos de $A$. lituratus, com os quais foram fornecidas 25 amostras de fezes. Na RPPN de Volta Velha foram capturados 90 indivíduos, totalizando 32 amostras de fezes. Os dados referentes à dieta nas duas localidades podem ser observados na figura 2. Em Superagüi, a dieta compreendeu 12\% de Ficus L. $(\mathrm{n}=3), 8 \%$ de Cecropia Loefl. $(\mathrm{n}=2), 12 \%$ de insetos $(\mathrm{n}=3)$ e $68 \%$ polpa indeterminada (amostras fecais com polpa mas sem sementes) $(\mathrm{n}=17)$. No total a dieta frugívora compreendeu $88 \%$ dos recursos utilizados. Na RPPN de Volta Velha a dieta foi composta por $25 \%$ de Ficus sp. $(\mathrm{n}=8), 21,9 \%$ de Solanum $\mathrm{L}$. $(\mathrm{n}=7), 6,25 \%$ de Cecropia sp. $(\mathrm{n}=2), 18,8 \%$ de insetos $(\mathrm{n}=6)$ e $28,1 \%(n=9)$ de polpa indeterminada. Dentre todos os recursos consumidos em Volta Velha observou-se que a dieta frugívora atingiu $81 \%$ do total. Foi constatada a utilização de

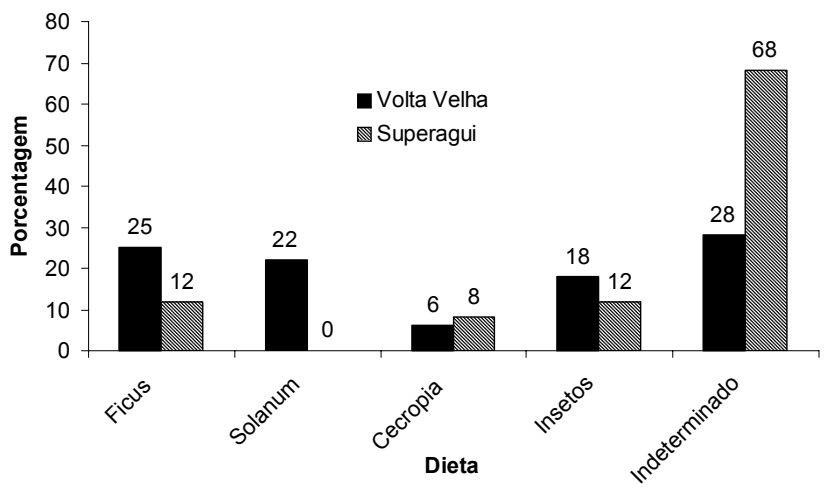

Figura 2. Dieta de Artibeus lituratus em duas localidades da região sul do Brasil, RPPN Volta Velha, Itapoá, Santa Catarina ( $n=32$ amostras fecais) e Parque Nacional de Superagüi, Guaraqueçaba, Paraná ( $n=25$ amostras fecais).

frutos com sementes grandes na dieta, com mais de $10 \mathrm{~mm}$ de comprimento e que não são engolidas. Esse foi o caso da coleta de animal com semente de Calophyllum brasiliense Cambess. aderido a rede de neblina. Tal fato comprova que o animal estaria transportando tal fruto para um poleiro de alimentação para posterior consumo.

\section{DISCUSSÃO}

A dieta desta espécie de morcego foi predominantemente frugívora (de 81 a $88 \%$ ) (Fig. 2), como já observado em outros estudos de $A$. lituratus (Fleming 1986, Galetti \& Morellato 1994, Zortea \& Chiarello 1994, Passos et al. 2003). Esta espécie é considerada um especialista em frutos de Cecropiaceae e Moraceae (Fleming 1986, Passos et al. 2003). O trabalho de Handley et al. (1991) também descreveu a importância das figueiras (Moraceae) para a dieta de A. jamaicensis Leach 1821, e indicou que Ficus insipida Willd. é seu alimento favorito. Apesar dessa preferência por Cecropiaceae e Moraceae, nos locais onde a densidade dessas plantas é baixa $A$. lituratus pode apresentar uma dieta mais generalista (Galetti \& Morellato 1994). Isso poderia ser um indicativo de uma grande plasticidade alimentar, permitindo uma adaptação às diferentes situações de oferta de alimento, ou seja, em condições de abundância de alimento a escolha recai sobre o tipo preferido, ou no caso de escassez de recursos, os animais acabam por utilizar uma estratégia alimentar generalista, consumindo uma variedade de espécies disponíveis.

Considerando isso, os dados demonstram que no PNS estas duas famílias (Cecropiaceae e Moraceae) representaram apenas $20 \%$ da dieta, enquanto na RPPN de Volta Velha estes recursos representam $31 \%$. Neste caso, a grande quantidade de amostras de fezes com polpa indeterminada, que impediu a identificação das espécies consumidas (sem sementes), de 28,1 a $68 \%$, pode estar relacionada ao consumo de frutos com se- 
mentes grandes que não são engolidas como no caso do guanandi Calophyllum brasiliense, ou ainda a uma maior importância de Cecropiaceae e Moraceae (caso parte dessas amostras sem semente fossem das respectivas famílias). Não foi constatado o consumo de folhas, mas este recurso pode compreender uma parcela considerável da dieta de $A$. lituratus em certas épocas do ano (Zortéa \& Mendes 1993, Zortéa \& Chiarello 1994).

Não foram observadas sementes de plantas de Solanum em Superagüi, o que pode indicar a ausência destas plantas para a área, mas também pode ser por questões de menor amostragem de fezes de $A$. lituratus na ilha quando comparadas a Volta Velha. As diferenças encontradas na dieta entre essas duas localidades podem estar relacionadas às diferenças florísticas entre a vegetação do PNS e a da RPPN de Volta Velha, mas maiores informações são também necessárias. Podem ainda indicar uma grande plasticidade dessa espécie de frugívoro, relacionada aos recursos alimentares consumidos em áreas distintas.

A utilização na dieta de frutos com sementes grandes (como no caso de Calophyllum brasiliense), pode ser indicativo de um importante recurso que não é amostrado por freqüência de ocorrência nos estudos de dieta por meio de fezes, mas apenas em estudos de dieta em poleiros de alimentação ou através de observações diretas (Galetti \& Morelato 1994, Sazima et al. 1994, TAdDei 1969, Zortéa \& Chiarello 1994). Algumas espécies de sementes grandes, que são consumidas e muitas vezes transportadas para poleiros de alimentação, mas que dificilmente constam de listas de espécies consumidas em estudos de dieta por meio de fezes, são os casos de Calophyllum brasiliense e Terminalia cattapa L. (Galetti \& Morellato 1994, Sazima et al. 1994), Mangifera indica L.(SAzIma et al. 1994, TADDEI 1969) e Andira Juss.(Zortea \& Chiarello 1994).

Entretanto, maiores informações sobre a ecologia alimentar dessa espécie de frugívoro são necessárias para a compreensão dos padrões encontrados em sua dieta. Mas deve-se destacar a importância de diferentes métodos de estudo de dieta (fezes, poleiros de alimentação e observações diretas) para uma análise mais abrangente da ecologia alimentar dessa importante espécie de morcego frugívoro. Isto se deve ao fato que cada método tem suas vantagens e permite a constatação de determinados alimentos utilizados em sua dieta.

\section{AGRADECIMENTOS}

Agradecimentos a Guadalupe Vivekananda, Diretora do Parque Nacional de Superagüi, e a Lucio Machado, proprietário da RPPN de Volta Velha, pela permissão para a realização deste estudo e pelo apoio logístico nas respectivas áreas. A Ives S. Arnoni e Laura Kremer pelo auxílio na preparação das sementes. Ao CNPq pelas bolsas aos autores.

\section{REFERÊNCIAS BIBLIOGRÁFICAS}

Fabian, M.E.; A.M. Rui \& K.P. De Oliveira. 1999. Geographical distribution of Phyllostomidae bats (Mammalia: Chiroptera) in Rio Grande do Sul (Brazil). Iheringia, Série Zoologia, Porto Alegre, (87): 143-156.

FARIA, D.M. DE. 1995. Os morcegos da Santa Genébra, p. 100106. In: P.L.C. Morellato \& H.F. Leitão-Filho (Eds). Ecologia e Preservação de uma floresta tropical urbana. Campinas, Universidade Estadual de Campinas, 136p.

Fleming, T.H. 1986. Opportunism versus specialization: evolution of feeding strategies in frugivorous bats, p. 105118. In: A. Estrada \& T.H. Fleming (Eds). Frugivores and seed dispersal. Dordrecht, W. Junk Puplisher, XIII+392p.

Fischer, W.A. \& E.A. Fischer. 1995. Comportamento social e reprodutivo do morcego-cara-branca, Artibeus lituratus, p. 106-110. In: P.L.C. Morellato \& H.F. Leitão-Filho (Eds). Ecologia e Preservação de uma floresta tropical urbana. Campinas, Universidade Estadual de Campinas, 136p.

Galetti, M. \& L.P.C. Morellato. 1994. Diet of the large fruiteating bat Artibeus lituratus in a forest fragment in Brazil. Mammalia, Paris, 58 (4): 661-665.

Handley Jr., C.O.; A.L. Gardner \& D.E. Wilson. 1991. Food Habits, p. 141-146. In: C.O. Handley JR.; D.E. Wilson \& A.L. GARDNER (Eds). Demography and natural history of the common fruit bat, Artibeus jamaicensis, on Barro Colorado island, Panamá. Washington, Smithsonian Contribution of Zoology, 180p.

Marinho-Filho, J. 1992. Mamíferos da Serra do Japi, p. 264-286. In: L.P.C. MoRellato (Ed.). História natural da Serra do Japi. Campinas, Universidade Estadual de Campinas, 321p.

Nowac, R.M. \& J.L. Paradiso. 1983. Walker's Mammals of the World. Baltimore, The Johns Hopkins University Press, vol. $1,4^{\text {th }}$ ed., XLIV $+568 \mathrm{p}$.

Passos, F.C.; W.R. Silva; W.A. Pedro \& M.R. Bonin. 2003. Frugivoria em Morcegos (Chiroptera, Phyllostomidae) no Parque Estadual Intervales, sudeste do Brasil. Revista Brasileira de Zoologia, Curitiba, 20(3): 511-517.

SAZima, I.; W.A. Fischer; M. SAZIma \& E.A. Fischer. 1994. The fruit bat Artibeus lituratus as a forest and city dweller. Ciência e Cultura, São Paulo, 46 (3): 164-168.

Rui, A.M.; M.E. Fabian \& J.O. Menegheti. 1999. Geographical distribution and morphological analysis of Artibeus lituratus Olfers and Artibeus fimbriatus Gray (Chiroptera, Phyllostomidae) in Rio Grande do Sul, Brazil. Revista Brasileira de Zoologia, Curitiba, 16 (2): 447-460.

TADDEI, V.A. 1969. Aspectos da biologia de Artibeus lituratus lituratus (Lichtenstein, 1823) (Chiroptera - Phyllostomidae). Ciência \& Cultura, São Paulo, 21 (2): 451-452.

ZortéA, M. \& A.G. Chiarello. 1994. Observations on the big fruit-eating bat, Artibeus lituratus, in an Urban Reserve of South-east Brazil. Mammalia, Paris, 58 (4): 665-670.

ZorTéa, M. \& S.L. Mendes. 1993. Folivory in the big fruit eating bat, Artibeus lituratus (Chiroptera: Phyllostomidae) in eastern Brazil. Journal of Tropical Ecology, Cambridge, 9: 117-120.

Recebido em 14.X.2003; aceito em 08.VII.2004. 